\title{
Radial velocities of dwarf spheroidal galaxies in the M 81 group ${ }^{\star}$
}

\author{
M. E. Sharina ${ }^{1,2}$, I. D. Karachentsev ${ }^{1}$, and A. N. Burenkov ${ }^{1,2}$ \\ 1 Special Astrophysical Observatory, Russian Academy of Sciences, N.Arkhyz, KChR, 369167, Russia \\ 2 Isaac Newton Institute, Chile, SAO Branch
}

Received 3 September 2001 / Accepted 5 October 2001

\begin{abstract}
Long-slit observations of 4 dwarf spheroidal galaxies in the M 81 group are presented. We have obtained the heliocentric velocity of a globular cluster candidate located near the center of DDO 78 to be $55 \pm 10 \mathrm{~km} \mathrm{~s}^{-1}$ by cross-correlation with template stars. We estimated a heliocentric radial velocity of $-116 \pm 21 \mathrm{~km} \mathrm{~s}^{-1}$ for an HII region seen in K 61. A red diffuse object near the K 64 center is found to be a remote galaxy with a heliocentric velocity of $+46530 \mathrm{~km} \mathrm{~s}^{-1}$.
\end{abstract}

Key words. galaxies: dwarf - galaxies: star clusters

\section{Introduction}

This work continues our study of dwarf spheroidal galaxies (dSphs) in the M 81 group. dSphs are diffuse spheroidal objects with central surface brightnesses of $\mu_{\mathrm{v}}(0) \gtrsim$ $22^{\mathrm{m}} / \square^{\prime \prime}$, absolute magnitudes $M_{\mathrm{V}} \gtrsim-14^{\mathrm{m}}$, and HI masses $M_{\mathrm{HI}} \lesssim 10^{5} M_{\odot}$ (Grebel 2000). Being among the faintest galaxies, dSphs are rather difficult to observe. As gas-poor galaxies, they are usually undetectable in the HI line. In the case of the M 81 group an additional observational difficulty arises, because of the group location in an area of the sky that is contaminated with dense Galactic HI emission (Appleton et al. 1993) and Galactic cirrus (Sandage 1976).

Almost half of the galaxies in the M 81 group are dSphs. There are DDO 71, DDO 78 and K 61, K 64 discovered many years ago by van den Bergh (1959) and Karachentseva (1968), as well as BK5N, BK6N, KK77, F8D1, FM1, and KKH57 found in more recent studies (Börngen \& Karachentseva 1982; Karachentsev 1994; Caldwell et al. 1998; Froebrich \& Meusinger 2000). Based on the presence of HII region, some blue stars and a probable presence of neutral gas Karachentsev et al. (2000) assumed K 61 to be dSph/dIrr transition type.

All these objects have been resolved into stars with Hubble Space Telescope Wide Field Planetary Camera 2 and confirmed as members of the M 81 group via tip of

Send offprint requests to: M. E. Sharina,

e-mail: sme@luna.sao.ru

* Based on observations collected with the $6 \mathrm{~m}$ telescope of the Special Astrophysical Observatory (SAO) of the Russian Academy of Sciences (RAS), operated under the financial support of the Science Department of Russia (registration number 01-43). red giant branch stars (Caldwell et al. 1998; Karachentsev et al. 2000; Karachentsev et al. 2001).

The HST images have allowed us to find globular cluster candidates in five galaxies of the M $81 \mathrm{dSphs}$ sample (Karachentsev et al. 2000). The images of four dSph galaxies in the M 81 group are presented in Fig. 1. Globular cluster candidates and central background galaxy in the case of K 64 are indicated by arrows. The images were obtained aboard the Hubble Space Telescope as part of a snapshot survey of nearby dwarf galaxy candidates (program GO 8192, PI: Seitzer) and produced by combining the two $600 \mathrm{~s}$ exposures taken through the $\mathrm{F} 606 \mathrm{~W}$ and F814W filters. The galaxies are centred in the WFC3 chip.

Basic photometric properties of globular cluster candidates from Karachentsev et al. (2000) are listed in Table 1. Its lines contain: (1) integrated apparent magnitude, (2) integrated color after correction for Galactic reddenning, $(3,4)$ angular and linear half-light radius, (5) the central surface brightness, and (6) integrated absolute magnitude. Globular clusters in dSphs are relatively bright objects. In most cases, spectroscopy of them is the only way to get radial velocities of parent galaxies. Data on radial velocities together with accurate distances for dSphs allow us to trace the structure and kinematics of the M 81 group in more detail.

\section{Observations and data reduction}

The observations were performed with the Long-slit spectrograph (Afanasiev et al. 1995) at the prime focus of the 6-m SAO (Russia) telescope during 4 nights in January 2001 at a seeing of $\sim 1^{\prime \prime}$ (see Table 2 for details). The long-slit $130^{\prime \prime}$ spectra were obtained with a CCD-detector having $1024 \times 1024$ pixels with $24 \times 24 \mu \mathrm{m}$ pixel size. 


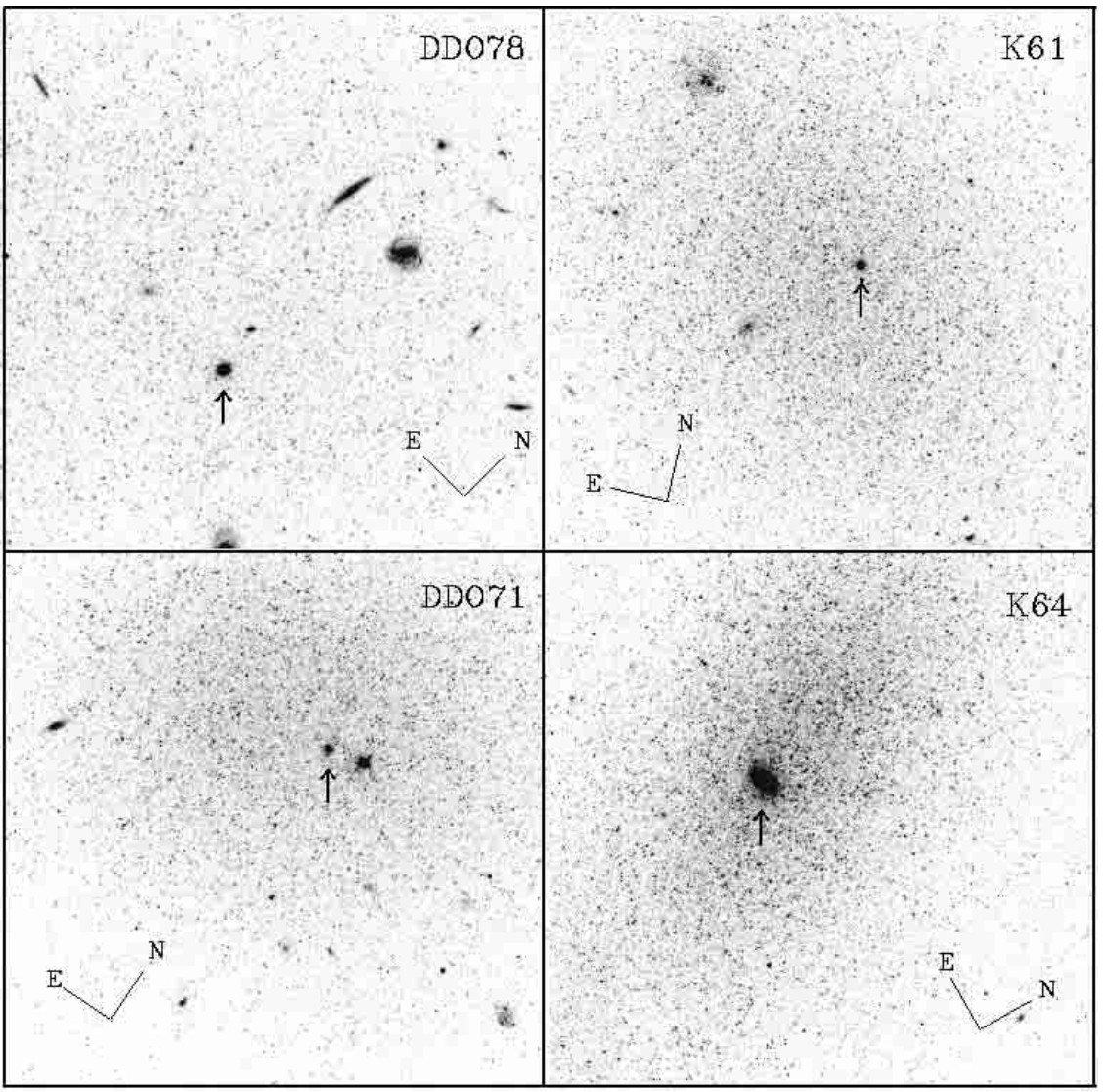

Fig. 1. WFPC2 images of four dSph galaxies in the M 81 group. Each galaxy is centered in the WF3 chip (WF3-FIX mode). The field size of each image is $1.3^{\prime}$. Globular cluster candidates and the central background galaxy in the case of $\mathrm{K} 64$ are indicated by arrows.
Table 1. Properties of globular cluster candidates from Karachentsev et al. (2000).

\begin{tabular}{lcccc}
\hline \hline Parameter & DDO 78 & K 61 & DDO 71 & K 64 \\
\hline$V_{\mathrm{T}}$ & 19.45 & 20.70 & 20.95 & $(19.5)$ \\
$(V-I)_{0}$ & 1.07 & 1.00 & 0.99 & $(1.44)$ \\
$\left.R(0.5 L),{ }^{\prime \prime}\right)$ & 0.30 & 0.20 & 0.26 & $(1.08)$ \\
$R(0.5 L), \mathrm{pc}$ & 5.3 & 3.6 & 4.6 & - \\
$\mu_{\mathrm{v}}(0)$ & 18.0 & 18.5 & 19.2 & $(19.6)$ \\
$M_{\mathrm{v}}$ & -8.48 & -7.37 & -7.17 & - \\
\hline \hline
\end{tabular}

K 64: the central object appears to be a background galaxy.

For all observations we used the grating of 651 grooves $/ \mathrm{mm}$ with corresponding dispersion of $2.4 \AA /$ pixel and a spectral resolution of 7-9 $\AA$, respectively. The slit positions (Table 2) were chosen to cross the star cluster candidate and any characteristic feature of the galaxy, like probable HII regions. The wavelength range is 4500$6900 \AA$. In all the cases the slit width was $2^{\prime \prime}$. The scale along the slit was $0.39^{\prime \prime} /$ pixel. The reference spectra of Ar-Ne-He lamp were recorded before and after each observation to provide wavelength calibration. For velocity calibration we have obtained long-slit spectra of five radial velocity standard stars (Barbier-Brossat \& Figon 2000) (see Table 2). The spectrophotometric standard star BD28 (Hamuy et al. 1994) was observed for flux calibration.

The data reduction was performed using the LONG package in MIDAS. The subsequent data analysis was also carried out in MIDAS. The primary data reduction included cosmic-ray removal, bias subtraction and flat-field correction. After wavelength calibration and sky subtraction, the spectra were corrected for atmospheric extinction and flux-calibrated. Then rows of every linearized twodimensional spectrum were summed in the spatial direction to yield a final one-dimensional spectrum. All individual exposures of the same object were then co-added to increase the signal-to-noise ratio. At last, the spectra of globular cluster candidates and template stars were divided by normalized continuum and wavelength rebinning was done linearly in $\ln \lambda$, as appropriate for the crosscorrelation analysis.

\section{Radial velocities}

Heliocentric radial velocities of the observed objects are summarized in Table 4.

\section{1. $D D O 78$}

DDO 78 has a very flat surface brightness profile with the central surface brightness $\mu_{\mathrm{v}}(0)=24.5^{\mathrm{m}} / \square^{\prime \prime}$, the exponential scale length $h=28^{\prime \prime}$ and the integrated magnitude 
Table 2. Observing log.

\begin{tabular}{|c|c|c|c|}
\hline Object & Date & Exposure & PA of the slit \\
\hline Globular cluster & 18.01 .2001 & $2 \times 1200 \mathrm{~s}$ & 49.0 \\
\hline candidate & 18.01 .2001 & $2 \times 2400$ & \\
\hline in DDO78 & 19.01 .2001 & $8 \times 1200$ & \\
\hline $\begin{array}{l}\text { HII region } \\
\text { and globular cluster } \\
\text { candidate in K } 61\end{array}$ & 18.01 .2001 & $2 \times 1200$ & $60^{\circ} 9$ \\
\hline $\begin{array}{l}\text { Background galaxy } \\
\text { projected onto K } 64\end{array}$ & 23.01 .2001 & $3 \times 1800$ & $60^{\circ} 0$ \\
\hline $\begin{array}{l}\text { Globular cluster } \\
\text { candidate in DDO } 71\end{array}$ & 23.01.2001 & $\begin{array}{l}2 \times 1800 \\
2 \times 1200\end{array}$ & 78.2 \\
\hline BF 10078 (F8 V) & 18.01 .2001 & $3 \times 10$ & \\
\hline BF 49601 (G8 V) & 18.01 .2001 & $3 \times 30$ & \\
\hline BF 13987 (F8 VI) & 18.01 .2001 & $3 \times 60$ & \\
\hline BF 18804 (G9 V) & 19.01.2001 & $2 \times 20$ & \\
\hline BF 18757 (G9 V) & 19.01.2001 & $2 \times 10$ & \\
\hline BD 28 & $\begin{array}{l}18.01 .2001 \\
19.01 .2001\end{array}$ & $\begin{array}{l}2 \times 90 \\
2 \times 150\end{array}$ & \\
\hline
\end{tabular}

$V_{\mathrm{t}}=15.1$ (Karachentsev et al. 2000). It is surprising that the galaxy contains the bright globular cluster candidate. The quality of the DDO 78 globular cluster candidate spectrum (Fig. 2) is adequate to derive its radial velocity. The spectra obtained independently in two observational nights have typically a signal-to-noise ratio of about 10 each.

The spectrum of the globular cluster in DDO 78 was cross-correlated with stellar template spectra using the method of Tonry \& Davis (1979). A procedure of MIDAS "XCORRELATE/IMAGE" correlates two onedimensional frames in pixel coordinates. The spectral resolution is approximately as twice as the expected internal velocity dispersion in the cluster, so we have not artificially broadened the template star spectra before the procedure of cross-correlation. The globular cluster spectrum was individually cross-correlated against the velocity templates observed in the corresponding night. Then the spectrum was divided into four parts. These parts were similarly cross-correlated against the corresponding spectral regions of the velocity templates. We also estimated the radial velocity by measuring redshifts of individual absorption lines. The latest method gave a larger rms error of radial velocity estimates when compared with the cross-correlation method. So the mean radial velocity from cross-correlation served as a final guess to the mean
Table 3. Measured heliocentric radial velocities of globular cluster (GC) in DD O78.

\begin{tabular}{lll}
\hline \hline Method & $\lambda \lambda \AA$ & $V_{\mathrm{h}}, \mathrm{km} \mathrm{s}^{-1}$ \\
\hline \hline Cross-correlation & & \\
\hline GC versus BF 13987 & $4700 \div 6700$ & +52 \\
& $4700 \div 5200$ & +56 \\
& $6200 \div 6700$ & +58 \\
GC versus BF 18804 & $4700 \div 6700$ & +61 \\
& $4700 \div 5200$ & +81 \\
GC versus BF 49601 & $6200 \div 6700$ & +46 \\
\hline \hline Individual Wavelength Shifts & $4700 \div 6700$ & +40 \\
\hline GC regarding to BF 18804 & $\mathrm{H} \alpha 6562.78$, & $+76 \pm 39$ \\
& $\mathrm{Fe}+\mathrm{CaI} 5270$, & \\
& $\mathrm{H} \beta 4861.33$ & \\
GC regarding to BF 49601 & $\mathrm{H} \alpha 6562.78$, & $+71 \pm 35$ \\
& $\mathrm{MgI} 5172.7,5183.6$, & \\
\hline \hline
\end{tabular}

Table 4. Heliocentric radial velocities of the observed objects.

\begin{tabular}{lc}
\hline \hline Object & $V_{\mathrm{h}}, \mathrm{km} \mathrm{s}^{-1}$ \\
\hline $\begin{array}{l}\text { Globular cluster } \\
\text { candidate in DD O78 }\end{array}$ & $55 \pm 10$ \\
HII region in K 61 & $116 \pm 21$ \\
$\begin{array}{l}\text { Background galaxy } \\
\text { projected onto K 64 }\end{array}$ & $46530 \pm 23$ \\
\hline \hline
\end{tabular}

wavelength-shift determination. The results and errors are summarized in Table 3.

Finaly, we conclude that the observed object is a true globular cluster belonging to DDO 78. Its mean heliocentric velocity is $+55 \pm 10 \mathrm{~km} \mathrm{~s}^{-1}$, where the error includes "internal" cross-correlation errors and statistical errors from different cross-correlation manners.

\section{2. $K 61$ and $D D O 71$}

The DDO 71 and K 61 globular cluster candidates have fainter apparent magnitudes: $V_{\mathrm{T}}=20.95$ and 20.70, respectively (Karachentsev et al. 2000). Being divided by normalized continuum, their spectra are presented in Fig. 3. The signal-to-noise ratio for them is poor and the cross-correlation peak vanishes into the noise. In the case of DDO 71 an additional difficulty arises, because the slit position was chosen to cross a foreground star. As a result, night sky lines have not been subtracted correctly. 

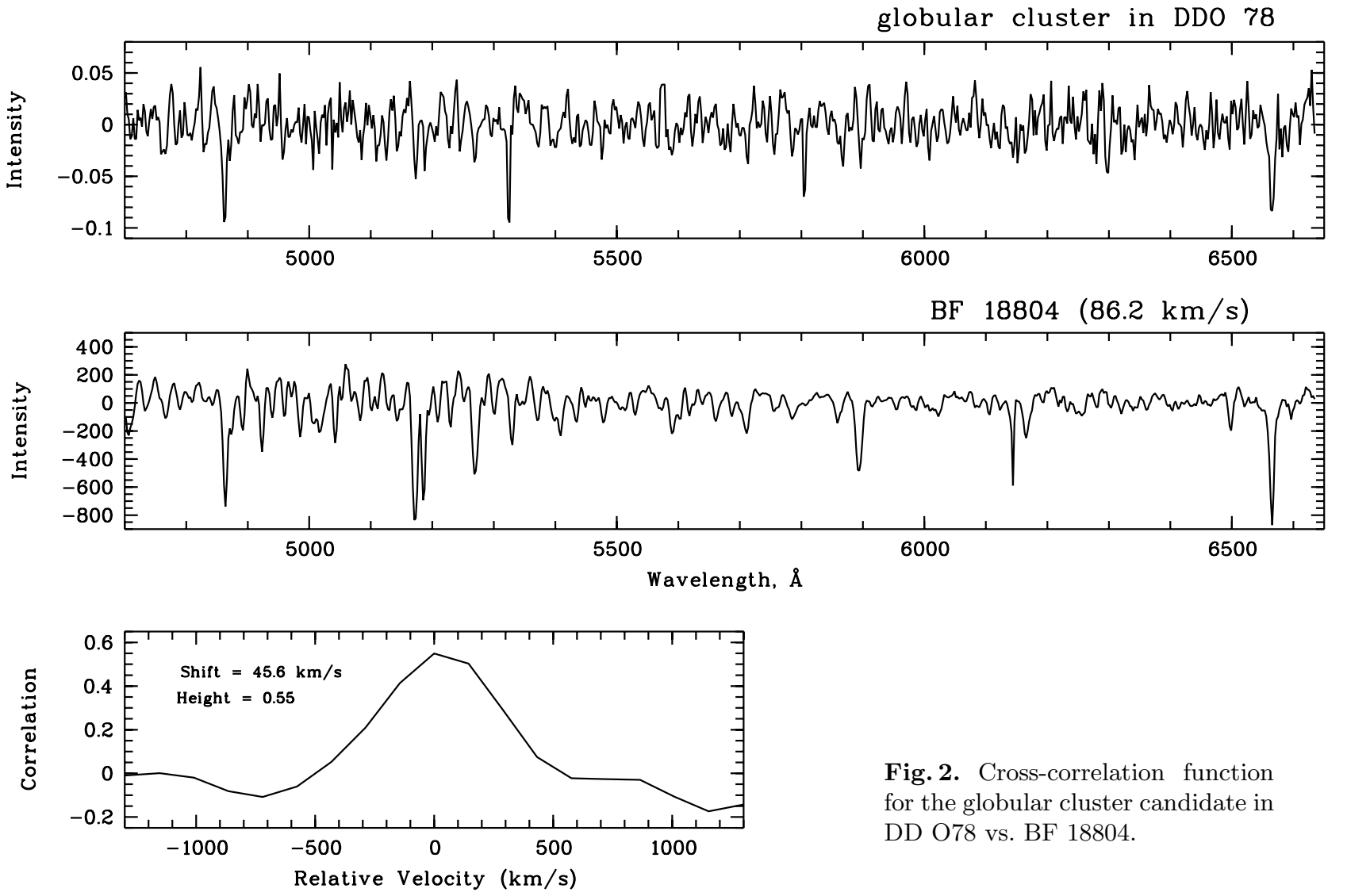

Fig. 2. Cross-correlation function for the globular cluster candidate in DD O78 vs. BF 18804.
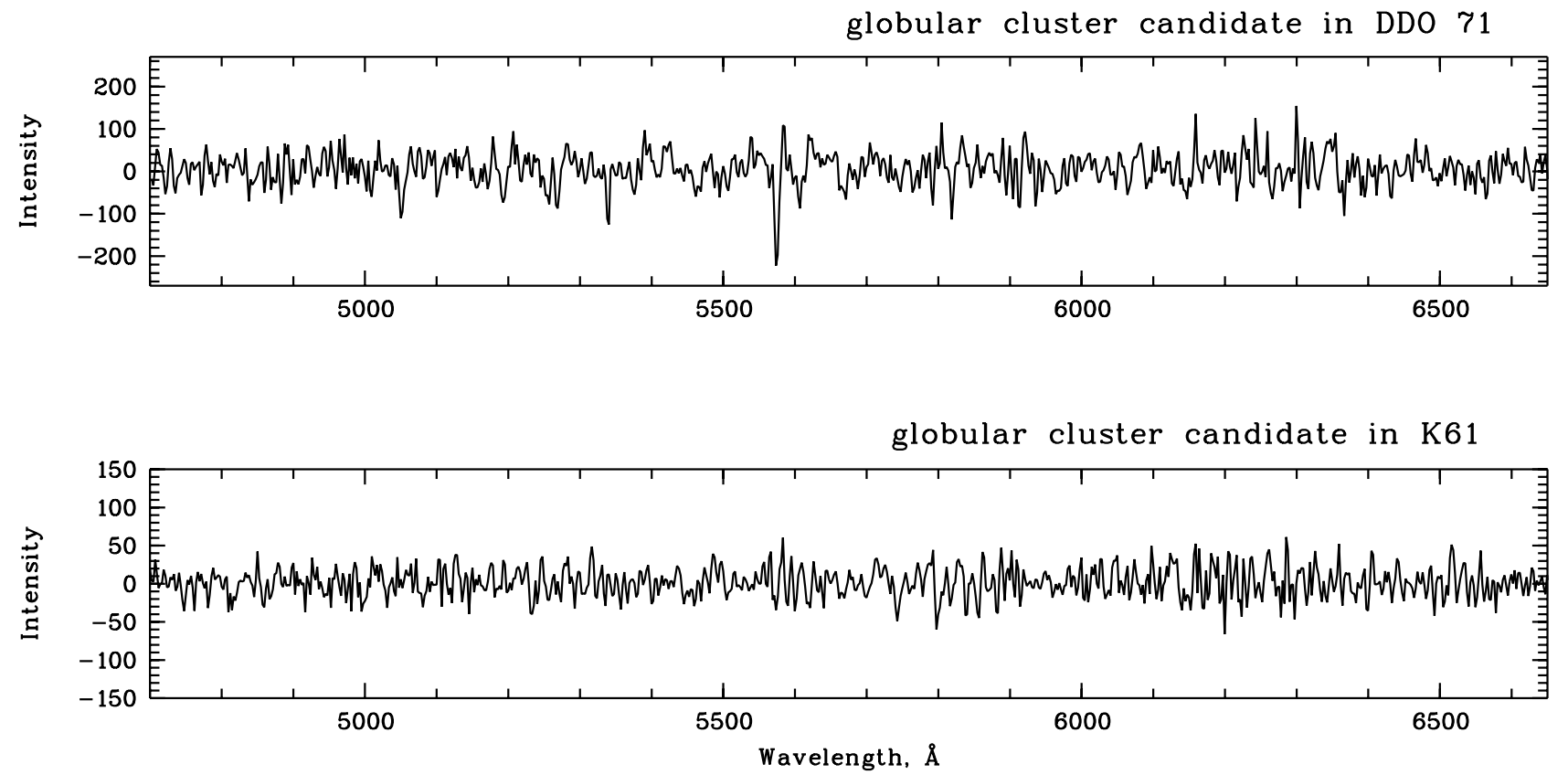

Fig. 3. Spectra of globular cluster candidates in K 61 and DD O71 divided by normalized continuum.

We have also obtained a spectrum of the HII region in $\mathrm{K} 61$ (Fig. 4). 4 Note, that K 61 is the brightest dSph galaxy in the M 81 group and the closest companion to M 81. Johnson et al. (1997) revealed a bright HII knot situated NE of the galaxy center. It shows high excitation emissions with a radial velocity of $-135 \pm 30 \mathrm{kms}^{-1}$.
Our velocity estimate agrees well with the previous data. The strongest lines in the spectrum, $\mathrm{H} \beta$, [OIII] $\lambda \lambda 4959,5007$ and $\mathrm{H} \alpha$, were used to measure the radial velocity of $\mathrm{K} 61$. As a result, the mean heliocentric velocity is $-116 \pm 21 \mathrm{~km} \mathrm{~s}^{-1}$, where the error is the standard deviation calculated from different lines. 


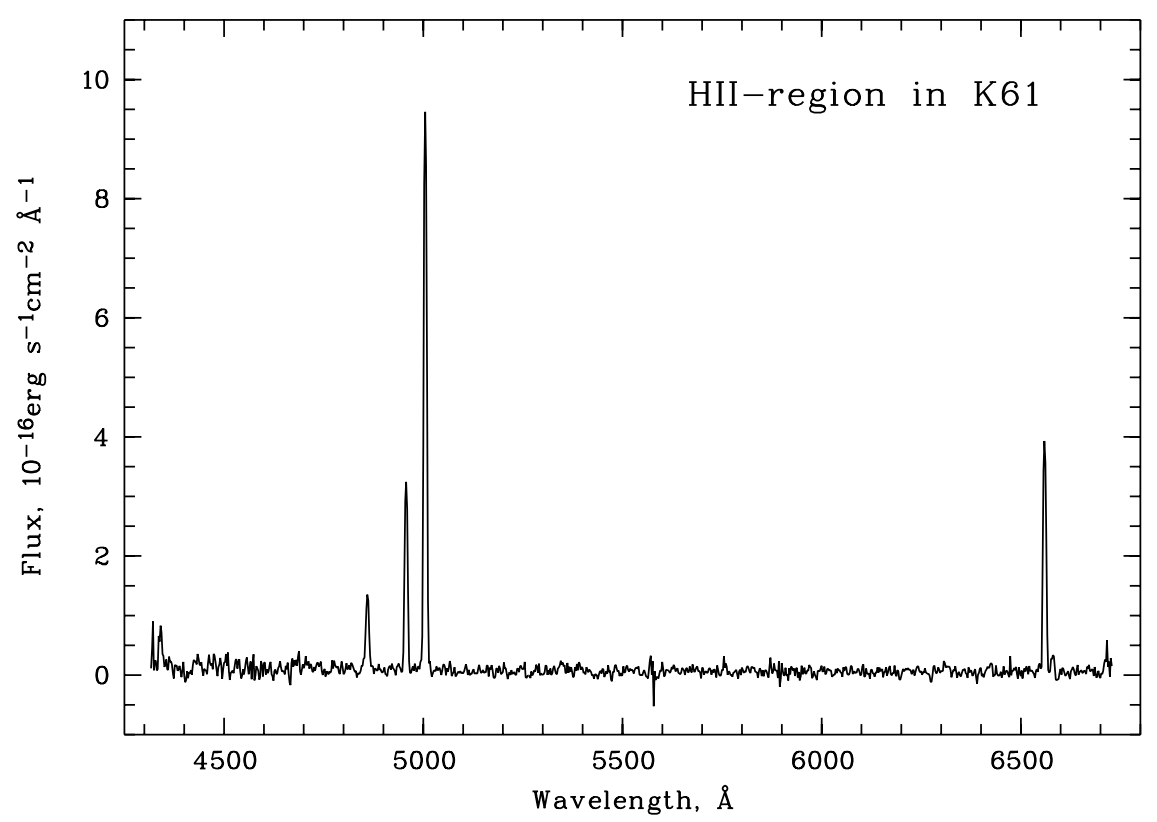

Fig. 4. Spectrum of HII region in K 61 .
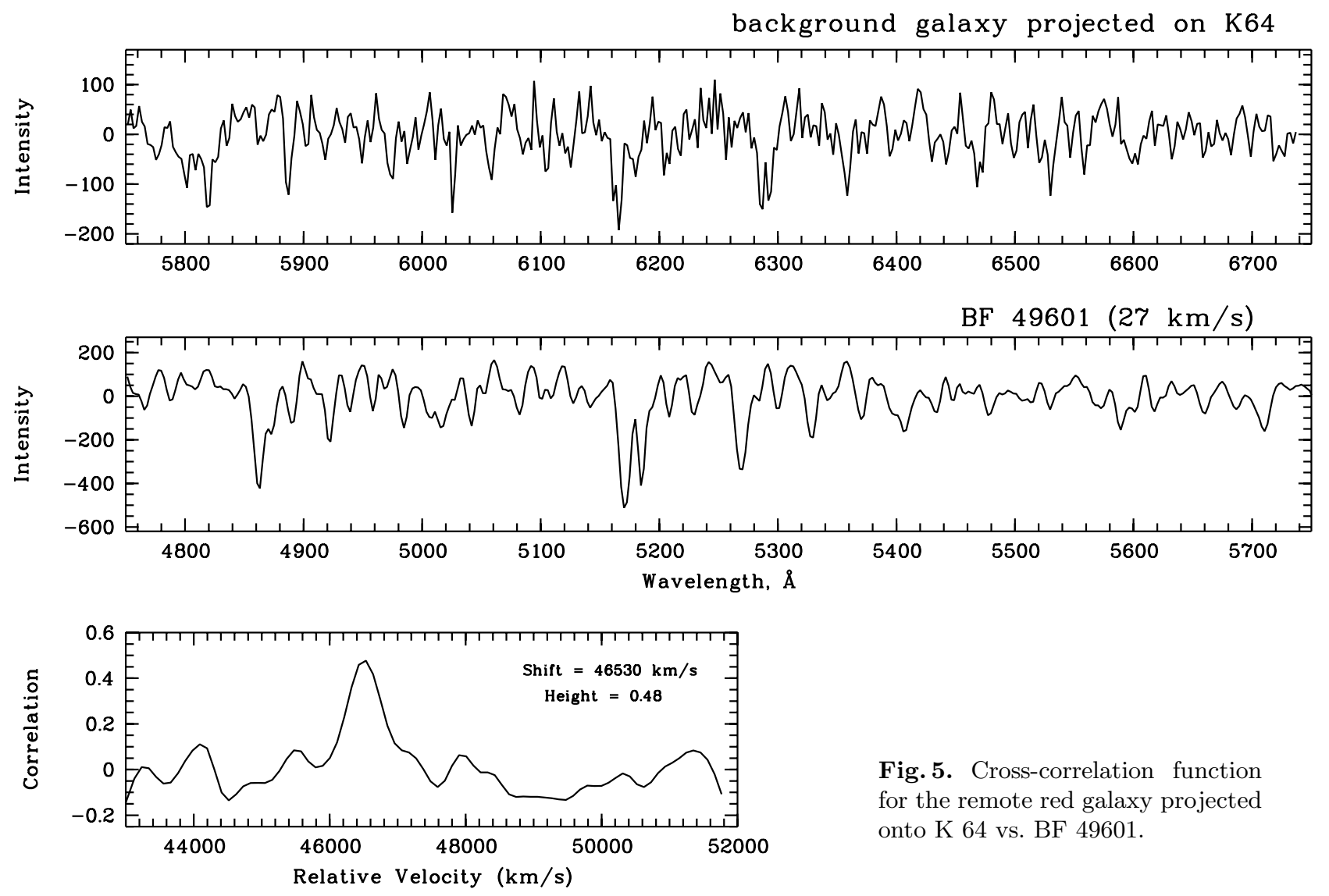

Fig. 5. Cross-correlation function for the remote red galaxy projected onto K 64 vs. BF 49601.

\subsection{K 64}

Binggeli \& Prugniel (1994) ascribed the central star-like object of K 64 as a "quasi-stellar nucleus". However, the large-scale HST images indicate this object to be a remote red galaxy (Karachentsev et al. 2000). Our spectral data confirm this conclusion. Figure 5 shows location of the measured cross-correlation peak. The spectra of the object and a template star are presented too. We have derived for the galaxy the mean heliocentric velocity $46530 \mathrm{~km} \mathrm{~s}^{-1}$.

Acknowledgements. The authors are grateful to A. V. Moiseev, and D. I. Makarov, who help us to master the methods of 
Long-slit reduction and cross-correlation. We thank A. G. Grebel, E. K. 2000, 33rd ESLAB Symp. on Star Formation Pramsky for help in observations.

This work has been partially supported by the DFG-RFBR grant 01-02-04006.

\section{References}

Appleton, P. N., Siqueira, P. R., \& Basart, J. P. 1993, AJ, 106, 1664 from the Small to the Large Scale, SP-445, ed. F. Favata, A. A. Kaas, \& A. Wilson (Noordwijk: ESA), 87

Hamuy, M., Suntzeff, N. B., Heathcote, S. R., et al. 1994, PASP, 106, 566-589

Johnson, R. A., Lawrence, A., Terlevich, R., \& Carter, D. 1997, MNRAS, 287, 333

Karachentsev, I. D. 1994, Astron. Astrophys. Trans., 6, 1

Karachentsev, I. D., Karachentseva, V. E., Dolphin, A. E., et al. 2000, A\&A, 363, 117

Afanasiev, V. L., Burenkov, A. N., Vlasyuk, V. V., \& Drabek, S. V. 1995, SAO RAS internal report, No. 234

Barbier-Brossat, M., \& Figon, P. 2000, A\&AS, 142, 217

Binggeli, B., \& Prugniel, P. 1994, La Lettre de l'OHP, No. 12

Börngen, F., \& Karachentseva, V. E. 1982, Astron. Nachr., 303,189

Karachentsev, I. D., Sharina, M. E., Dolphin, A. E., et al. 2001, A\&A, 375, 359

Karachentseva, V. E., \& Karachentsev, I. D. 1998, A\&AS, 127, 409

Karachentseva, V. E., Karachentsev, I. D., \& Börngen, F. 1985, A\&AS, 60, 213

Caldwell, N., Armandroff, T. E., da Costa, G. S., \& Seitzer, P. 1998, AJ, 115, 535

Froebrich, D, \& Meusinger, H. 2000, A\&AS, 145, 229

Sandage, A. R. 1976, AJ, 81, 964

Tonry, J., Davis, M. 1979, AJ, 84, 1511

van den Bergh, S. 1959, Publ. of DDO, vol. II, No. 5, 147 